\title{
METAL MESH COUPLERS USING EVANESCENT WAVES AT MILLIMETER AND SUBMILLIMETER WAVELENGTHS
}

\author{
Jongsuck Bae ${ }^{\dagger}$, Jung-Chih Chiao ${ }^{\ddagger}$, Koji Mizuno ${ }^{\dagger}$, and David B. Rutledgeł \\ †Research Institute of Electrical Communication, Tohoku University, \\ 2-1-1 Katahira, Aoba-ku, Sendai 980-77, Japan
${ }^{\dagger}$ Photodynamics Research Center, The Institute of Physical and Chemical Research, \\ 19-1399 Aza-Koeji, Nagamachi, Aoba-ku, Sendai 980, Japan \\ $\ddagger$ Department of Electrical Engineering, California Institute of Technology, \\ Pasadena, California 91125, USA
}

\begin{abstract}
A metal mesh evanescent wave coupler which makes use of coupling of evanescent waves between a metal mesh and a tuning dielectric plate, has been developed as a quasioptical component for millimeter and submillimeter wavelengths. The coupling coefficient of the capacitive evanescent wave coupler can be changed more than $70 \%$ by moving the silicon plate only $60 \mu \mathrm{m}$ at around $53 \mathrm{GHz}$. The transmission properties of the evanescent wave couplers depending on the mesh period and the silicon plate thickness have been studied.
\end{abstract}

\section{INTRODUCTION}

Fabry-Perot interferometers have been widely used as tunable optical couplers or filters at millimeter and submillimeter wavelengths. Fabry-Perot type optical couplers consist of two reflectors and make use of the interference between propagating waves in the couplers to change the coupling coefficient. Consequently, a large and rapid variation of the coupling coefficient with frequency results in a narrow bandwidth. A metal mesh evanescent wave (MEW) coupler has been presented as a quasi-optical component that ameliorates the trade-off between bandwidth and coupling coefficient of Fabry-Perot type couplers [1] [2].

The MEW-coupler consists of an inductive or a capacitive metal mesh and a flat dielectric plate placed close to the surface of the mesh (Fig. 1), and makes use of evanescent wave coupling to vary the reflectance and transmittance. The evanescent waves are induced by an incident wave on the mesh, and decay quickly away from the mesh, normally less than $\lambda / 20$ from the surface [3]. Therefore, in contrast to a Fabry-Perot interferometer, the coupling coefficient of the MEW-coupler can be significantly changed by small adjustments of spacing between the mesh and the di- electric plate. In principle, the MEW-coupler can also have a wider bandwidth because the transmission properties of the couplers depend primarily on the mesh parameters $(g$, $2 a$ and $t$ shown in Fig. 1). In this paper, the theoretical and experimental results are reported to show the dependence of the basic transmission properties on the mesh period and the movable dielectric plate thickness for the MEW-couplers.

\section{EVANESCENT WAVE COUPLING EFFECT}

The MEW-coupler consists of a silicon plate and an inductive or a capacitive metal mesh mounted on a quartz plate. The quartz plate has a refractive index of 2.12 and a

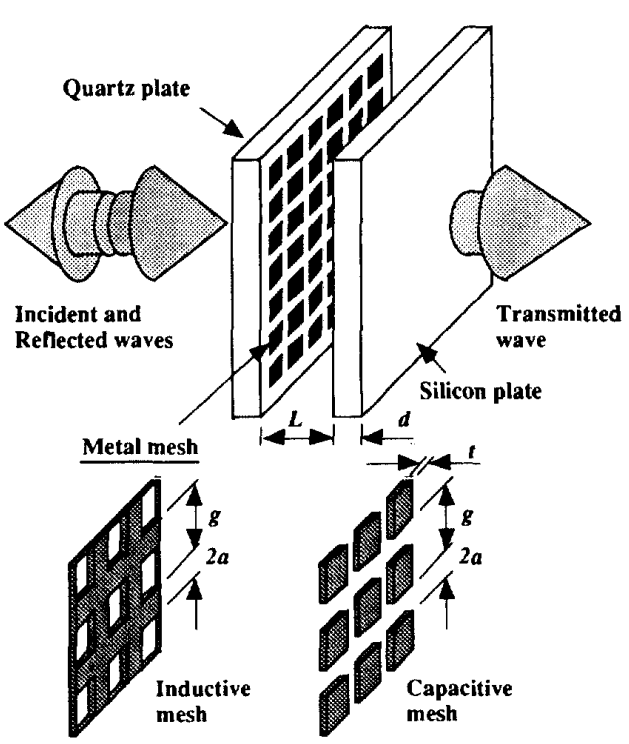

Fig. 1 Configuration of the MEW-coupler. 


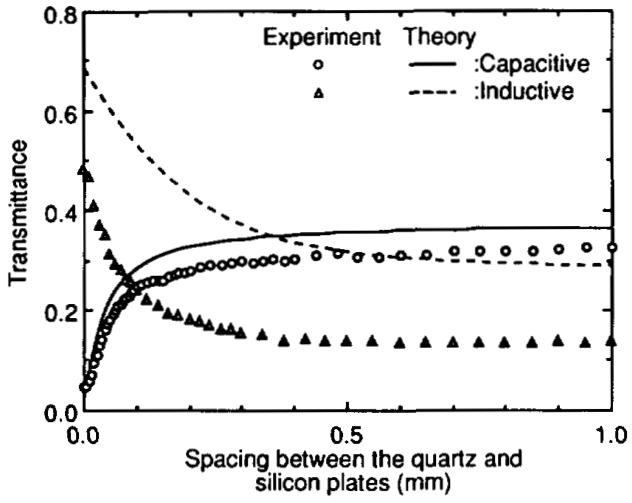

Fig. 2 Transmittance of the MEW-couplers with $(g, 2 a)=$ $(2.12,0.86) \mathrm{mm}$ at $44 \mathrm{GHz}$

thickness of $2 \mathrm{~mm}$. The silicon plates have a refractive index of 3.42 and have different thicknesses, $d$, of 1,2 , and 3 $\mathrm{mm}$. The refractive indices of the quartz and silicon plates have been determined by measuring their transmissions in the frequencies range between $40 \mathrm{GHz}$ and $60 \mathrm{GHz}$.

The reflectance $\left(\mid S_{1 l^{2}}{ }^{2}\right)$ and transmittance $\left(\left|S_{2,}\right|^{2}\right)$ of the MEW-couplers with different mesh parameters and different silicon plate thicknesses have been estimated using the Method of Moments (MOM) and corroborated by the Hewlett-Packard High Frequency Structure Simulator (HFSS) in the $40-65 \mathrm{GHz}$ frequency range [4]. Experimental measurements have been done to verify the theoretical predictions. The measurement setup includes an HP85106C millimeter-wave network analyzer, transmitting and receiving horns and a MEW-coupler. More details about the theoretical and experimental conditions are described in reference [4].

Fig. 2 shows the measured transmittance of the MEWcouplers with meshes of $(g, 2 a)=(2.12,0.86) \mathrm{mm}$ as a function of the spacing $L$ between the quartz and silicon plates at $44 \mathrm{GHz}$. The silicon plate thickness is $1 \mathrm{~mm}$. At this frequency, the thickness of the silicon plate is about half of the dielectric wavelength in silicon, hence the Fabry-Perot etalon effect between the metal mesh and the silicon plate should disappear and then we can measure the evanescent wave coupling effect more precisely. The transmittance of the capacitive coupler increases when the spacing increases while the one of the inductive coupler decreases. The results show that the evanescent wave coupling effect occurs at the spacing less than $0.3 \mathrm{~mm}$ in both the capacitive and inductive couplers.

Fig. 3 shows the measured transmittances of the capacitive MEW-couplers with $(g, 2 a)=(1.7,0.69) \mathrm{mm}$ and $d$ $=1 \mathrm{~mm}$ as a function of frequency from 40 to $60 \mathrm{GHz}$. The experimental results show that the coupling coefficient of

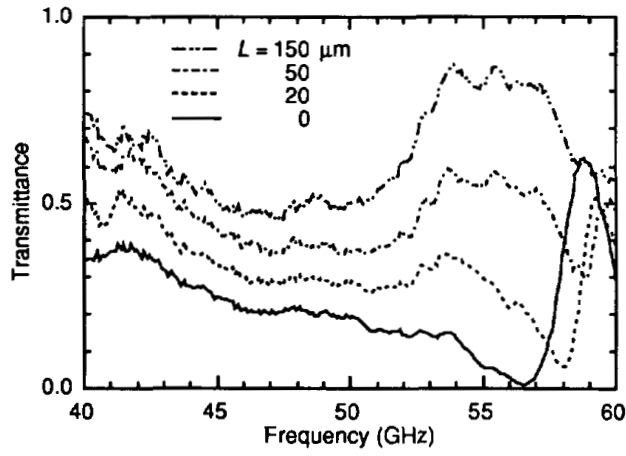

Fig. 3 Measured transmittance of the capacitive MEW-coupler with $(g, 2 a)=(1.7,0.69) \mathrm{mm}$ as a function of frequency for different spacing $L$. The silicon plate with a thickness of $1 \mathrm{~mm}$ has been used in this experiment.

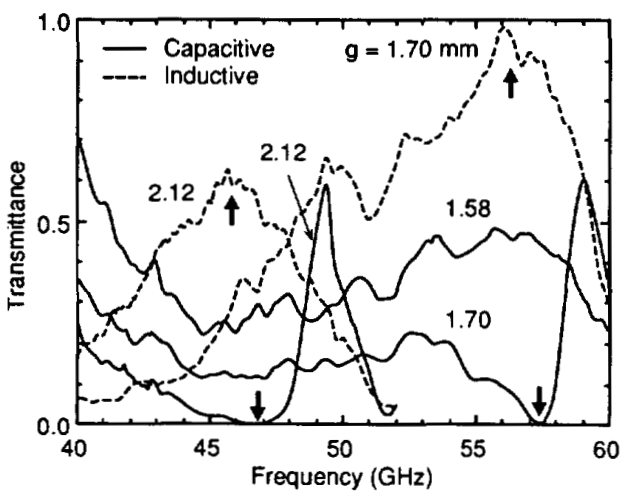

Fig 4 Measured transmittance of the MEW-couplers with different mesh parameters: $(g, 2 a)=(1.58,0.65),(1.7$, $0.69)$, and $(2.12,0.86) \mathrm{mm}$ as a function of frequency at $L=0$

the capacitive coupler can be changed more than $70 \%$ for a variation of $L=0.15 \mathrm{~mm}$ at around $56 \mathrm{GHz}$. For the same variation of $L$, the changes of the transmittance more than $25 \%$ have been obtained at the frequencies between 40 $\mathrm{GHz}$ and $58 \mathrm{GHz}$. Those experimental results show that the capacitive MEW-coupler has a wideband transmission property.

\section{RESONANT EFFECT}

The conventional mesh theory has shown that a resonant frequency of a metal mesh on a dielectric material is determined by $c /(g n)$, where $c$ is the light velocity, $g$ is the mesh period and $n$ is the refractive index of the dielectric plate; and that diffraction occurs above the resonant frequency [5] [6]. Since diffraction causes transmission loss, the opera- 

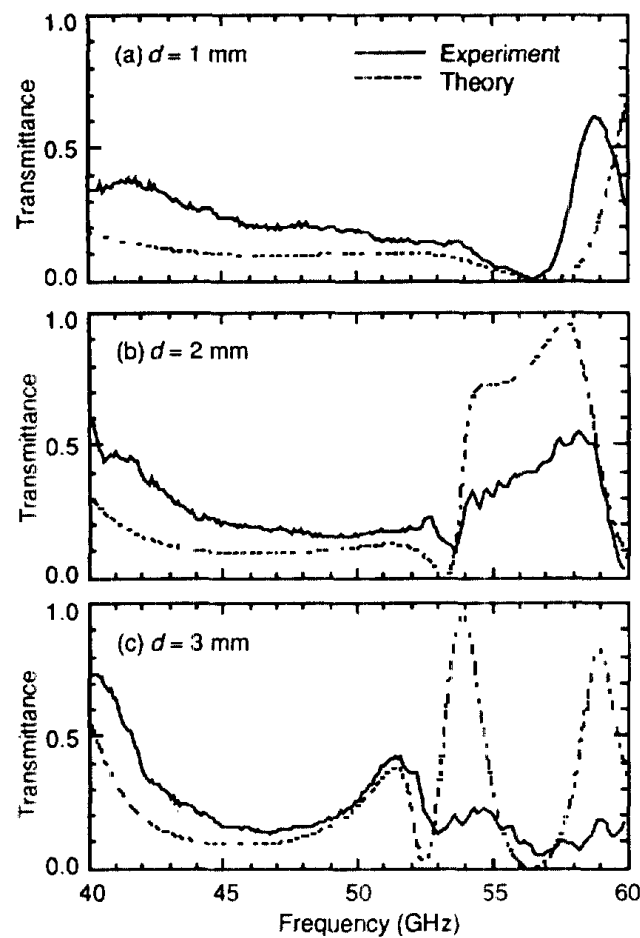

Fig. 5 Transmittance of the capacitive MEW-couplers with metal meshes of $(g, 2 a)=(1.7,0.69) \mathrm{mm}$ and different silicon plates as a function of frequency at $L=0$.

tion of the coupler is limited below the resonant frequency. Therefore, in order to design a MEW-coupler, we need to determine the resonant frequency.

\section{Dependence on the mesh period}

Fig. 4 shows the measured transmittances of the capacitive and inductive couplers as a function of frequency for different mesh periods at $L=0$. The thickness of the silicon plate is $1 \mathrm{~mm}$. In Fig. 4, the arrow marks indicate dips and peaks in the transmittance curves caused by the resonance. From the experimental results, it is seen that the resonant frequencies are about $57 \mathrm{GHz}$ and $46 \mathrm{GHz}$ for $g=$ $1.7 \mathrm{~nm}$ and $2.12 \mathrm{~mm}$, respectively. The relationship between the measured resonant frequencies and the mesh periods follows the conventional mesh theory. However, both results show that the effective refractive index of silicon is about 3.1. This value is smaller than the refractive index of silicon, 3.42 , determined by measuring the transmission of the $1 \mathrm{~mm}$ thick silicon plate at the frequencies between 40 $\mathrm{GH} z$ and $60 \mathrm{GHz}$. This discrepancy of the refractive indices results from the thin silicon plate thickness $(1 \mathrm{~mm})$ for the

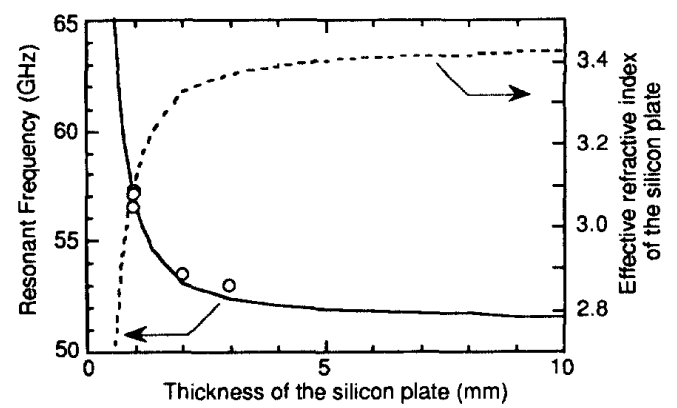

Fig. 6 Calculated resonant frequencies of the capacitive MEW-couplers with $(g, 2 a)=(1.7,0.69) \mathrm{mm}$ as a function of the silicon plate thickness. The effective refractive index of silicon was estimated from the calculated resonant frequencies. The circles in the figure indicate the measured resonant frequencies.

evanescent waves in this frequency range.

\section{Dependence on the silicon plate thickness}

The transmittances of the capacitive MEW-coupler with $(g, 2 a)=(1.7,0.69) \mathrm{mm}$ have been measured at $L=0$ for different silicon plates with thicknesses, $d$, of 1,2 , and 3 $\mathrm{mm}$ (Fig. 5). The resonant frequencies, where the dips in the transmission curves appear, are $56.5,53.5$, and $53 \mathrm{GHz}$ for $d=1,2$, and $3 \mathrm{~mm}$, respectively. Theory has well predicted these resonant frequencies within an accuracy of 1 $\%$. Above the resonant frequencies, the measured transmittances are largely different from the calculated values though their shapes are still similar. This discrepancy between the calculated and measured transmittances could be caused by diffraction in the measurements, because the diffraction loss of the coupler has not been taken into account in the theoretical simulation. Those results verify our assumption of the relationship between the resonant frequency and the silicon plate thickness.

Fig. 6 shows the calculated resonant frequencies of the capacitive MEW-coupler with $(g, 2 a)=(1.7,0.69) \mathrm{mm}$ as a function of the silicon plate thickness $d$. The measured resonant frequencies are also indicated in the same figure. The effective refractive indices of the silicon plate were estimated from the resonant frequencies. When the silicon plate thickness increases, the effective refractive index of silicon increases exponentially approaching to the actual value, 3.42 . With a thickness of $3.4 \mathrm{~mm}$, which is twice of the mesh period $g=1.7 \mathrm{~mm}$, the effective refractive index of silicon is greater than 3.37 which is $98.5 \%$ of the actual value. Therefore, we conclude that the silicon plate should have a thickness at least two times greater than the mesh period to reach the actual refractive index of silicon which has been measured for the propagating waves. When the 
thickness of the silicon plate goes to zero, the resonant frequency increases and approaches to the frequency determined by the refractive index of quartz, 83.2 GHz. Therefore, the effective refractive index of silicon in the coupler can be changed between 2.12 and 3.42 by adjusting the thickness of the silicon plate.

The resonance does not only determine the maximum operation frequency of the coupler, but also changes the transmission property. Fig. 7 shows the measured transmittance of the capacitive MEW-couplers with the silicon plates thickness, $d$, of 1,2 , and $3 \mathrm{~mm}$ as a function of the spacing between the quartz and silicon plates. The transmittances were measured at the resonant frequency corresponding to the respective thickness. The mesh parameters are $(g, 2 a)=(1.7,0.69) \mathrm{mm}$. From the experimental results, it is found that the transmittances of the couplers can be changed more than $70 \%$ for a variation of $L$ less than 120 $\mu \mathrm{m}, 60 \mu \mathrm{m}$, and $40 \mu \mathrm{m}$ for the silicon plates with $d=1,2$, and $3 \mathrm{~mm}$, respectively. In the experimental results shown in Fig. 3, the coupling coefficient only change $45 \%$ for a variation of $L=50 \mu \mathrm{m}$ at $53 \mathrm{GHz}$ in the capacitive MEWcoupler with a $1 \mathrm{~mm}$ thick silicon plate. Therefore, changing the silicon plate thickness from 1 to $3 \mathrm{~mm}$ can increase the variation of the coupling coefficient by 1.5 times. These experimental results show that the maximum variation of the coupling coefficient can be reached by adjusting the silicon plate thickness at any frequency between two resonant frequencies $51.6 \mathrm{GHz}$, which happens when the thickness of the silicon plate is infinite, and $83.2 \mathrm{GHz}$, which is determined by the quartz substrate.

\section{CONCLUSION}

Transmission properties of the evanescent wave (MEW) couplers have been investigated. From the theoretical and experimental results in the 40 to $60 \mathrm{GHz}$ range, it is found that the effective refractive index of the silicon plate changes exponentially from 3.42 to 2.12 when the thickness of the silicon plate decreases. This fact shows that the transmission properties of the MEW-coupler can be changed by adjusting the plate thickness of silicon. In a capacitive MEW-coupler, changing the silicon plate thickness from 1 $\mathrm{mm}$ to $3 \mathrm{~mm}$, the coupling coefficient can be increased from $45 \%$ to $70 \%$ for a variation of the spacing between the quartz and silicon plates less than $\lambda / 100$ at around 53 $\mathrm{GHz}$. These results show the dependence of transmission properties on the silicon plate thickness and on the mesh period. They also provide design information for the MEWcouplers.

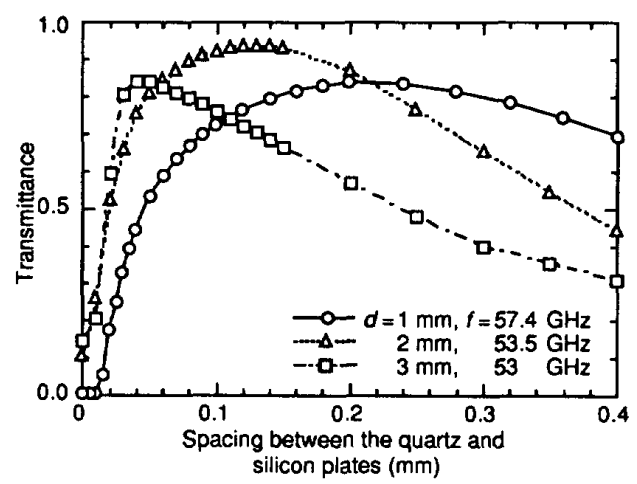

Fig. 7 Measured transmittance of the capacitive MEW-couplers with $(g, 2 a)=(1.7,0.69) \mathrm{mm}$ and $d=1,2$, and 3 $\mathrm{mm}$ at the resonant frequencies.

\section{ACKNOWLEDGMENTS}

The authors would like to acknowledge Dr. Richard Compton at Cornell University for helpful discussion. We would like to express our gratitude to Y. Aburakawa, T. Fujii, and M. Miyajima at Tohoku University for fabricating the metal mesh and the other experimental apparatus. We also thank Michael DeLisio at CalTech for assistance with the theoretical simulations. We appreciate the support from the Jet Propulsion Laboratory.

\section{REFERENCES}

[1] J. Bae, J. C. Chiao, K. Mizuno, and D. B. Rutledge, "Metal Mesh Couplers Using Optical Tunneling Effect at Millimeter and Submillimeter Wavelengths," The Digest of 1994 IEEE MTT-S Int. Microwave Symp., San Diego, pp. 787$790,1994$.

[2] J. C. Chiao, J. Bae, K. Mizuno, and D. B. Rutledge, "Metal Mesh Couplers Using Evanescent Waves at Millimeter and Submillimeter Wavelengths," The Digest of 19th Int. Conf. Infrared and Millimeter Waves, Sendai, Japan, pp. 365-366, 1994.

[3] Z. S. Agronovich, V. A. Marchenko, and V. P. Shestopalov, "The Diffraction of Electromagnetic Waves from Plane Metallic Lattices," Sov. Phys. Tech. Phys., vol. 7, pp. 277$286,1962$.

[4] J. Bae, J. C. Chiao, K. Mizuno, and D. B. Rutledge, "Metal Mesh Couplers Using Evanescent Waves at Millimeter and Submillimeter Wavelengths," Int. Journal of Infrared and Millimeter Waves, vol. 6, no, 2,1995.

[5] R. Compton, L. Whitbourn, and R. McPhedran, "Strip grating at a dielectric interface and application of Babinet's principle," Appl. Opt., vol. 23, pp. 3236-3242, 1984.

[6] D. H. Dawes, R. C. McPhedran, and L. B. Whitbourn, "Thin capacitive meshes on a dielectric boundary: theory and experiment," Appl. Opt., vol. 28, pp. 3498-3510, 1989. 